\section{SCIENTIFIC BOOKSHOP}

H. K. LEWIS can supply'works in all branches of Pure and Applied Science. Catalogues on request. Please state interests.

\section{SCIENTIFIC LENDING LIBRARY}

Annual Subscription from $\mathbf{\mathbf { 7 } . 5 0}$. (Available in U.K. only)

Reduced rates for multiple subscriptions.

\section{Prospectus post tree on request.}

Quarterly List of New Books and new editions added to the Library sent post free to subscribers regularly.

\section{H. K. LEWIS \& Co. Ltd. 136 GOWEA STREET, LONDON, WC1E 6BS}

\section{Telophon: 01-387 4282 \\ Tolegrams: "Publitcav",}

London, WC1E 68S."

\section{Circle No. 15 on Reader Enquiry Card.}

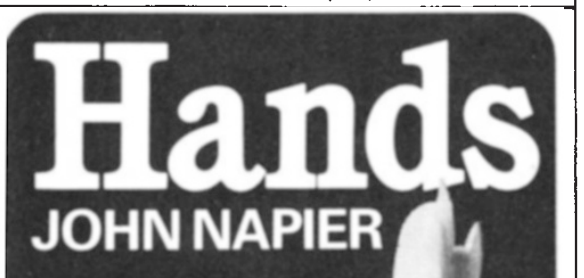

A popular account of the human hand; this book is written in a style accessible to the layman, as well as to a wide variety of scientists. The result is a fascinating amalgam of anatomical, biological and historical observation and comment.

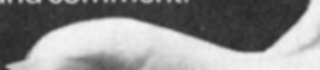

'Dr Napier Is

one of the small

but notable band

of British scientists

equally well known and

distinguished for their scien-

tific work... and for their ability

to popularise their work without distorting it:

DesmondMorris George $\mathbf{E 1 2 . 5 0}$ Alleng 2 Jnwin

Circle No. 26 on Reader Enquiry Card.

\title{
Clouds in the interstellar medium
}

\section{B. E. Turner}

Giant Molecular Clouds in the Galaxy. Edited by P. M. Solomon and M. G. Edmunds. Pp. 300. (Pergamon: 1980.) $£ 18, \$ 41$.

THE distribution and physical properties of the neutral interstellar gas in our galaxy have been major subjects of investigation since the discovery of interstellar atomic hydrogen in 1951. With the discovery 12 years ago of interstellar molecules as a new investigative tool, these studies have been extended to the molecular component with the undertaking of two major surveys of galactic carbon monoxide (CO) in 1975. The present volume is a collection of papers on this and related subjects as they stood in mid-1977 at the Third Gregynog Astrophysics Workshop, held at the University of Wales.

As with much of astrophysics, our perceptions of the interstellar medium vary on a timescale rather short compared with that of the publication of this volume. Even the title suffers as a result. It refers to one of two main topics covered, namely the properties of the molecular cloud ensemble that is generally agreed to comprise by mass at least $75 \%$ of the total gas of the galaxy interior to the Sun. The principal question is whether the cloud ensemble is dominated by relatively few 'giant molecular clouds' (GMCs) or by a larger number of smaller clouds. Curiously, the title belies the fact that only one of the two survey groups (Solomon and his collaborators) subscribe to the notion of GMCs, while the other group (Burton and Gordon) find that smaller clouds dominate. Loosely, GMCs may be defined as having masses in excess of $10^{5} M_{\odot}$ (diameters $>40 \mathrm{pc}$ ) as distinct from the many smaller clouds $\left(10^{2}-10^{4}\right.$ $M_{\odot}$ ) seen optically near the Sun. Whether or not GMCs dominate (the question is one of interpretation, not of differences in survey data by the two groups) is important to our understanding of galactic structure, because relatively few GMCs affect the distribution of stars and their motions much more than does the same quantity of gas residing in more numerous, smaller clouds. In the past year, extensive modelling of galactic cloud distributions by Liszt and Burton has shown that the observations are consistent with an ensemble of numerous, smaller clouds with at most only about $4 \%$ of the clouds by number being GMCs. Although the title of the book may therefore describe only the minority component, it must be emphasized that GMCs are known observationally, the dozen or so examples being discussed in the first two papers.

Star formation, and its association with molecular clouds, is the other principal topic of this book. Where stars may be directly observed, it has been clear for some time that massive $O B$ stars form in or at the edges of the larger molecular clouds (mass of $10^{4} M \odot$ or larger) while only low-mass stars (including $T$ Tauris) form in small clouds. Observational examples of each of these cases are given in two papers. Other articles discuss various mechanisms for the onset of massive star formation, such as density wave implosions, sequential formation by $\mathrm{O}$ star radiation, supernova inducement, cloud-cloud collisions and so on. Doubts about the applicability of any of these mechanisms to at least one object (CRL961) are discussed by L. Blitz, who contributes much of the observational material in this book not available in the open literature. The probable association of $\mathrm{OH}$ and $\mathrm{H}_{2} \mathrm{O}$ masers with massive protostellar objects is set forth in three observational papers, and the relation of HII regions and molecular clouds is the topic of two papers. On the theoretical side, magnetic fields (the bete noire of any model of star formation) are discussed with originality if inconclusion in two papers.

In addition to the above two topics, this volume includes several articles on peripherally related subjects: some outdated work on the galactic centre (two papers); two works on highly specific aspects of interstellar chemistry (on nitrogen chemistry and interstellar biochemicals - the latter is notably speculative and has appeared in journals several times); and finally, even an entertaining paper on the search for planetary systems.

As might be judged from such a plethora of topics, the book is not intended, nor does it succeed, as a synthesis of any aspect of interstellar clouds. Unfortunately, there are no discussion sessions which might have tied the various viewpoints together; thus the forum of a workshop is not reflected in the book. The introduction by the editors serves as a (rather one-sided) overview, but not more.

The belated publication of this volume has limited the usefulness of much of it; no fewer than 21 of the 31 papers, and parts of some others, have appeared in journals. Nevertheless, some valuable material has not been published elsewhere. Examples are the beautiful colour maps of the Solomon et al. CO survey, the large-scale $\mathrm{CO}$ maps of the Cygnus $\mathrm{X}$, Taurus and Perseus regions in the paper by Blitz, and several of the theoretical discussions.

The book is handsomely produced on high-quality paper, with fine illustrations and a well-thought-out index. Overall, it is certainly to be recommended as a reference book for institutional libraries, and at the price it may well find a place in the personal collections of serious students of the interstellar medium. It is less suitable for a more general audience.

B.E. Turner is at the National Radio Astronomy Cbservatory, Charlottesville, Virginia. 